\title{
Time-Dependent Change in Baroreflex Control Capacity of Arterial Pressure by Pentobarbital Anesthesia in Rabbits
}

\author{
Shin-ichiro KATSUDA ${ }^{1)}$, Hidefumi WAKI'), Tadanori NAGAYAMA ${ }^{11}$, Masao YAMASAKI'), \\ Hirotaka O-ISHI ${ }^{1)}$, Kiyoaki KATAHIRA2), and Tsuyoshi SHIMIZU1)
}

\author{
${ }^{1)}$ Department of Physiology and 2)Experimental Animal Research Center, Fukushima Medical \\ University School of Medicine, 1 Hikari-ga-oka, Fukushima 960-1295, Japan
}

\begin{abstract}
The present study is designed to investigate the time-dependent effect of pentobarbital anesthesia on the baroreflex arterial pressure $(A P)$ control system in rabbits. The overall AP control capacity of the baroreflex system was assessed with mean arterial pressure (MAP) responses to the rapid mild hemorrhage $(2 \mathrm{ml} / \mathrm{kg}$ body weight) and an overall open-loop gain $(G)$ of the system. The $G$ value was determined by means of the following formula: $G=\Delta A P_{/} \Delta A P_{S^{-1}}$, where $\triangle A P_{1}$ is an immediate $M A P$ fall and $\triangle A P_{S}$ a steady-state fall after the rapid hemorrhage. Prior to the experiment, two catheters for $A P$ measurement and hemorrhage were chronically in-dwelt in the aortic arch via the left subclavian and left common carotid arteries, respectively. Control mean arterial pressure averaged for $30 \mathrm{sec}$ before the rapid hemorrhage (CMAP), $\triangle A P_{I}$ and $\triangle A P_{S}$ significantly increased and reached the maximal value at $14 \min (C A M P: p<0.01)$ and $28 \min \left(\triangle A P_{1}\right.$ : $p<0.01$ and $\left.\triangle A P_{S}: p<0.01\right)$ after the intravenous injection of sodium pentobarbital in a 25.0 $\mathrm{mg} / \mathrm{kg}$ dose, respectively. These values gradually decreased in the course of time and tended to recover to near the preanesthetic level at 77-98 min after the anesthesia. The $G$ value significantly decreased from 7.3 in the conscious state to 1.5 at 28 min after the anesthesia $(p<0.001)$, gradually increased with lapse of time and recovered to near the preanesthetic level at 77-98 min after the anesthesia. No significant difference in $G$ was observed between in the conscious and anesthetized states beyond $70 \mathrm{~min}$ after the anesthesia ( $p>0.05)$. These findings suggest that pentobarbital sodium exerts a timedependent inhibitory effect on the baroreflex system but does not significantly affect the overall AP control capacity of the baroreflex system itself at least 70 min after the intravenous administration at a dose of $25.0 \mathrm{mg} / \mathrm{kg}$.
\end{abstract}

Key words: baroreflex system, open-loop gain, pentobarbital sodium, rabbit

(Received 28 October 1999 / Accepted 2 February 2000)

Address corresponding: S. Katsuda, Department of Physiology, Fukushima Medical University School of Medicine, 1 Hikari-ga-oka, Fukushima 960-1295, Japan 


\section{Introduction}

Pentobarbital sodium has often been used for cardiovascular study in veterinary and pre clinical medicine. It is known that pentobarbital anesthesia can affect cardiovascular function, whereas it decreased fluctuation in cardiovascular parameters i.e., arterial pressure (AP) and heart rate (HR), and so forth, establishing stable experimental conditions.

Pentobarbital anesthesia has been indicated to play a major part in the autonomic nervous system $[3,5,16$, $19,21,22]$, which is responsible for modifying the sensitivity of baroreflex AP or HR control [1, 2, 8, 16]. Zimpfer, et al. [24] reported that pentobarbital sodium reduced the capacity to restore hypotension due to hemorrhage. On the other hand, Hosomi and Sagawa [8] and we [13] demonstrated that pentobarbital sodium did not affect the baroreflex AP control capacity after hemorrhage. The reason for the discrepancy in the effect of pentobarbital anesthesia on the control capacity of the baroreflex AP control system, although associated with many factors, remains to be clarified.

The effect of anesthetics in general gradually diminishes after administration, accompanied with a decrease in the concentration in plasma, though it depends on dosage, species, age and other conditions. The AP level tends to return to the preanesthetic level with time after the anesthesia. The time from the administration of anesthetic to starting the experiment has not been clearly reported in most studies. This would be one of the important factors in the disparity among investigations in the effect of pentobarbital anesthesia on the circulatory system.

In the present study, we investigate the time-dependent effect of pentobarbital sodium on the baroreflex AP control capacity and compare it with that under conscious conditions in rabbits. The control capacity of the baroreflex system was assessed by the mean arterial pressure (MAP) responses to rapid mild hemorrhage and the overall open-loop gain $(\mathrm{G})$ of the system.

\section{Materials and Methods}

Animals: Fourteen normal Japanese white conventional rabbits (body weight; $3.1 \pm 0.4 \mathrm{~kg}$ and age; 18.5 \pm 3.8 months old, mean \pm SD, Fujii Animals, Co., Hyogo, Japan) were used in the present study. They were bred in an individual wire cage with a commercial pellet diet (ORC-4, Oriental Yeast, Co., Ltd., Japan) at $100 \mathrm{~g} /$ day in a room with the temperature at 22$25^{\circ} \mathrm{C}$ and relative humidity at $50-60 \%$.

Experimental protocol: A schematic arrangement of a laboratory animal and setup are shown in Fig. 1. The rabbits were anesthetized with an intravenous injection of pentobarbital sodium at a dose of $30.0 \mathrm{mg} / \mathrm{kg}$. Two large bore catheters (1.2 mm, I.D.) for AP measurement and rapid mild hemorrhage were chronically placed into the aortic arch through the left subclavian and the left common carotid arteries, respectively. The other ends of both catheters were subcutaneously tunneled to the back and connected to stop cocks. The catheters were filled with heparin (Novo heparin, Kodama, Co., Ltd., Japan) every day at a dose of 500 $\mathrm{U} / \mathrm{kg}$ to prevent blood coagulation. Several days after the catheterization, we confirmed that the rabbits were active and in a good state of health. They were transferred from the previous cage to the small experiment cage. Two hours was allowed to acclimate the animal to the experiment environment. MAP in the aortic arch was measured with a catheter, transducer (MPU-0.5A, Nihon Kohden Kogyo, Co., Ltd., Japan) and preamplifier (AP-6200, Nihon Kohden Kogyo Co., Ltd., Japan) through a low-pass filter with a time constant of $2 \mathrm{sec}$. A small amount of blood ( $2 \mathrm{~m} / \mathrm{kg}$ body weight) was withdrawn from the aortic arch into a syringe with a catheter within 1-2 sec as quickly as possible under

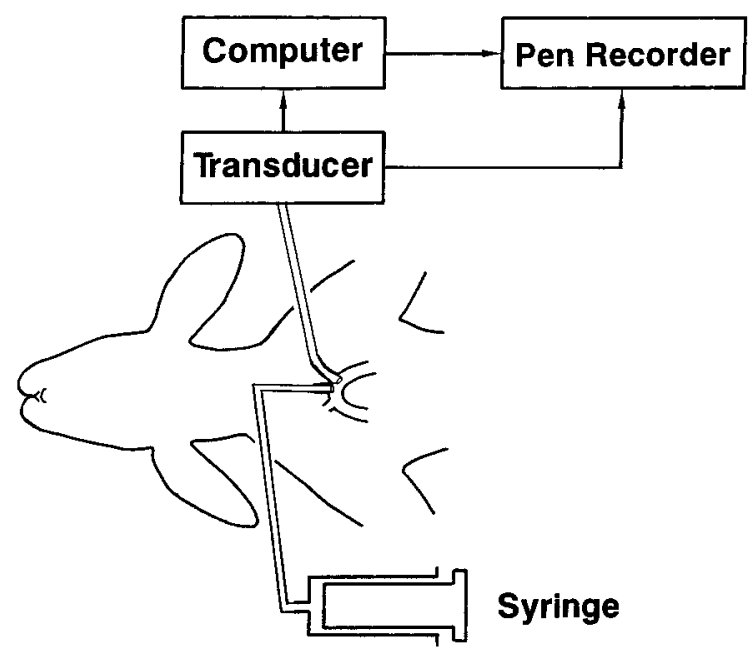

Fig. 1. Schematic arrangement of laboratory animal and experimental set-up. 
conscious conditions. MAP response to the rapid mild hemorrhage was recorded with a pen-recorder and simultaneously fed into a computer (JEC-7E, JEOL Ltd., Japan) through an analogue-to-digital converter at intervals of $100 \mathrm{msec}$ for $30 \mathrm{sec}$ before and $120 \mathrm{sec}$ after the hemorrhage. The shed blood was slowly re-infused via the hemorrhage catheter $3 \mathrm{~min}$ after the rapid hemorrhage. This hemorrhage-infusion procedure was repeated 8 times for each animal. After the hemorrhage experiment in the conscious state, the rabbits were anesthetized again with an intravenous administration of pentobarbital sodium at a dose of $25.0 \mathrm{mg} / \mathrm{kg}$ to evaluate the effect of pentobarbital sodium on the baroreflex AP control system. Rapid mild hemorrhage was started $7 \mathrm{~min}$ after the anesthesia at intervals of 7 min for 98 min.

Data analysis: The computer-stored strings of MAP responses under conscious and anesthetized (77-98 min after the onset of anesthesia) conditions were averaged 4 and 8 times to improve the signal-to-noise ratio due to changes in posture, and other factors, respectively.

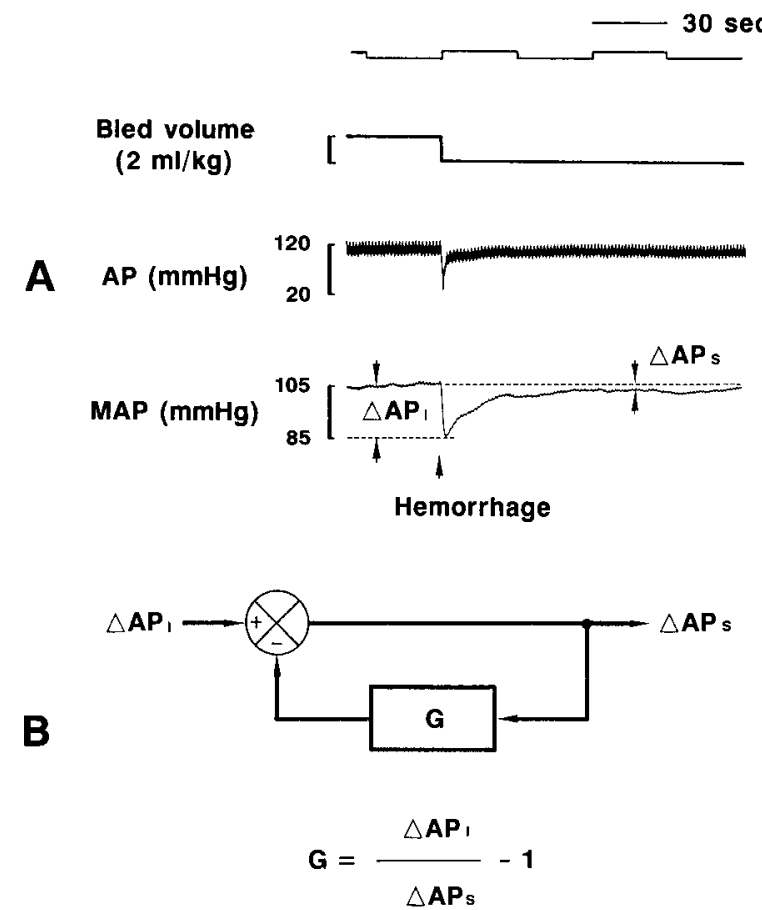

Fig. 2. Example of MAP response to rapid mild hemorrhage (A) and schematic illustration of the feedback AP control of the baroreflex system (B). The open-loop gain $(\mathrm{G})$ of the system was determined as $\Delta \mathrm{AP}_{\mathrm{I}} / \Delta \mathrm{AP}_{\mathrm{S}}-1$, where $\Delta \mathrm{AP}_{\mathrm{I}}$ was an immediate MAP fall after rapid hemorrhage and $\Delta \mathrm{AP}_{\mathrm{S}}$ a steady-state MAP fall 1-2 min after hemorrhage.
Control mean arterial pressure (CMAP) was averaged in MAP for $30 \mathrm{sec}$ before the rapid hemorrhage. Fig. 2 shows a typical MAP response to the rapid mild hemorrhage (A) and determination of $G$ of the overall baroreflex AP control system (B). $\Delta \mathrm{AP}_{\mathrm{I}}$ was taken as the peak of instantaneous MAP fall immediately after the rapid hemorrhage and $\Delta \mathrm{AP}_{\mathrm{S}}$ was measured as a steady-state fall observed 1-2 min after the hemorrhage. $\mathrm{G}$ was assessed on the basis of the linear control theory $[6,7,9,15]$ by the following formula: $\mathrm{G}=\Delta \mathrm{AP}_{\mathrm{I}} / \Delta \mathrm{AP}_{\mathrm{S}}{ }^{-1}$. The data were analyzed by Tukey's multi-comparison test for preanesthetic and postanesthetic conditions at each time after confirming significant difference by analysis of variance.

\section{Results}

Figure 3 shows example recordings of MAP response to the rapid mild hemorrhage before and after pento-

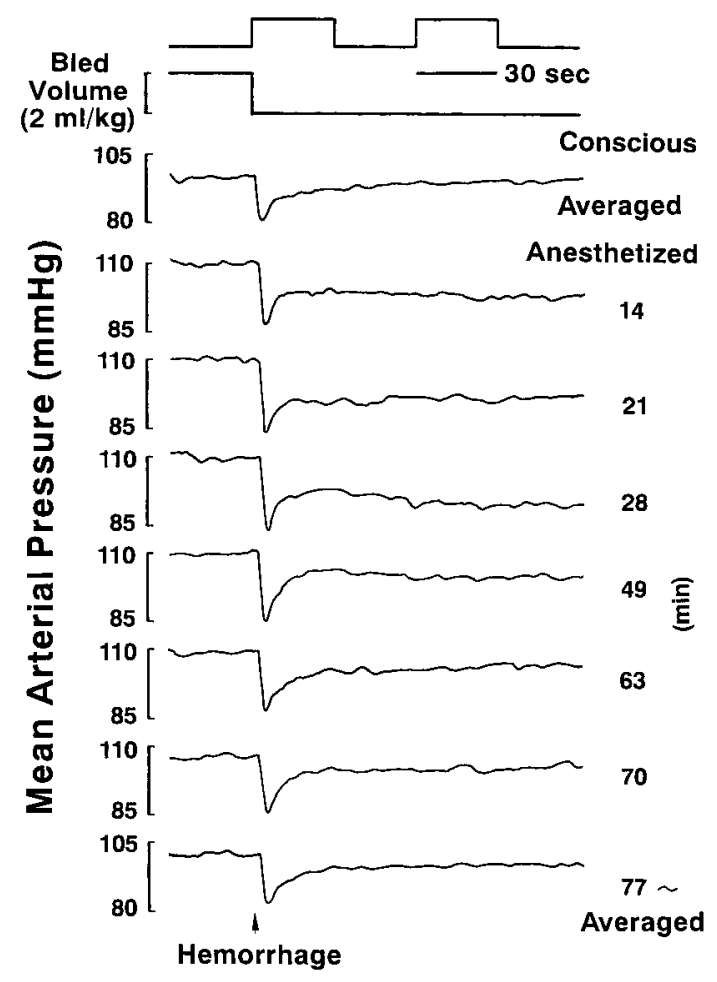

Fig. 3. Typical MAP responses to rapid mild hemorrhage in the conscious and anesthetized states. Pentobarbital sodium was intravenously administered at a dose of $25.0 \mathrm{mg} / \mathrm{kg}$. The responses averaged 8 and 4 times in the conscious and anesthetized (77-98 min after the anesthesia) states to improve the signal to noise ratio, respectively. 
barbital anesthesia. In the response under conscious conditions, MAP transiently fell immediately after the rapid hemorrhage and thereafter recovered to near the prehemorrhage level with a slight steady-state fall. Immediate MAP fall $\left(\triangle \mathrm{AP}_{\mathrm{I}}\right)$ considerably increased from 14 to $63 \mathrm{~min}$ after the anesthesia. The steady-state MAP fall $\left(\Delta \mathrm{AP}_{\mathrm{S}}\right)$ increased after the anesthesia, reached its maximum at $28 \mathrm{~min}$ after the anesthesia and thereafter gradually decreased.

Figure 4 shows changes in CMAP plotted as a function of time. CMAP was suddenly increased from 90.8 on average in the conscious state to $102.4 \mathrm{mmHg}$ at 14 min $(\mathrm{p}<0.01)$, gradually decreased with time, and almost returned near the preanesthetic level at 77-98 $\mathrm{min}$ after the anesthesia. No significant difference was observed at 77-98 min after the anesthesia ( $p>0.05$ ). Time-dependent changes in $\Delta \mathrm{AP}_{\mathrm{I}}$ and $\Delta \mathrm{AP}_{\mathrm{S}}$ induced by pentobarbital anesthesia are shown in Figs. 5 and 6, respectively. The values for $\Delta \mathrm{AP}_{\mathrm{I}}$ and $\Delta \mathrm{AP}_{\mathrm{S}}$ were 9.2 and $1.1 \mathrm{mmHg}$ under conscious conditions, respectively. $\Delta \mathrm{AP}_{\mathrm{I}}$ significantly increased immediately after the anesthesia, reached the maximal value of $18.9 \mathrm{mmHg}$ at $28 \min (\mathrm{p}<0.01)$, remained high and gradually decreased from $56 \mathrm{~min}$ after the anesthesia. There was no significant difference in $\Delta \mathrm{AP}_{\mathrm{I}}$ at $77-98 \mathrm{~min}$ after the anesthesia, though the value was relatively high in comparison with that under preanesthetic conditions. $\Delta \mathrm{AP}_{\mathrm{S}}$ rose to $8.3 \mathrm{mmHg}$ at $28 \mathrm{~min}$ after the anesthesia and thereafter gradually decreased to around the preanesthetic level. No significant difference in $\Delta \mathrm{AP}_{\mathrm{S}}$ between in the conscious and anesthetized states was observed from $63 \mathrm{~min}$ after theanesthesia.

Figure 7 depicts a change in $\mathrm{G}$ with time. G significantly decreased from 7.3 in the conscious state to 1.5 on average at $28 \mathrm{~min}$ after the anesthesia $(\mathrm{p}<0.001)$ and gradually recovered with time. G returned to near the preanesthetic level at 77-98 min after the anesthesia. There was no significant difference between $G$ in the conscious state and that in the anesthetized state from 70 min after the anesthesia $(p>0.05)$.

\section{Discussion}

There is a volume of research literature on the effects of pentobarbital anesthesia on cardiovascular function. Although most investigators have observed

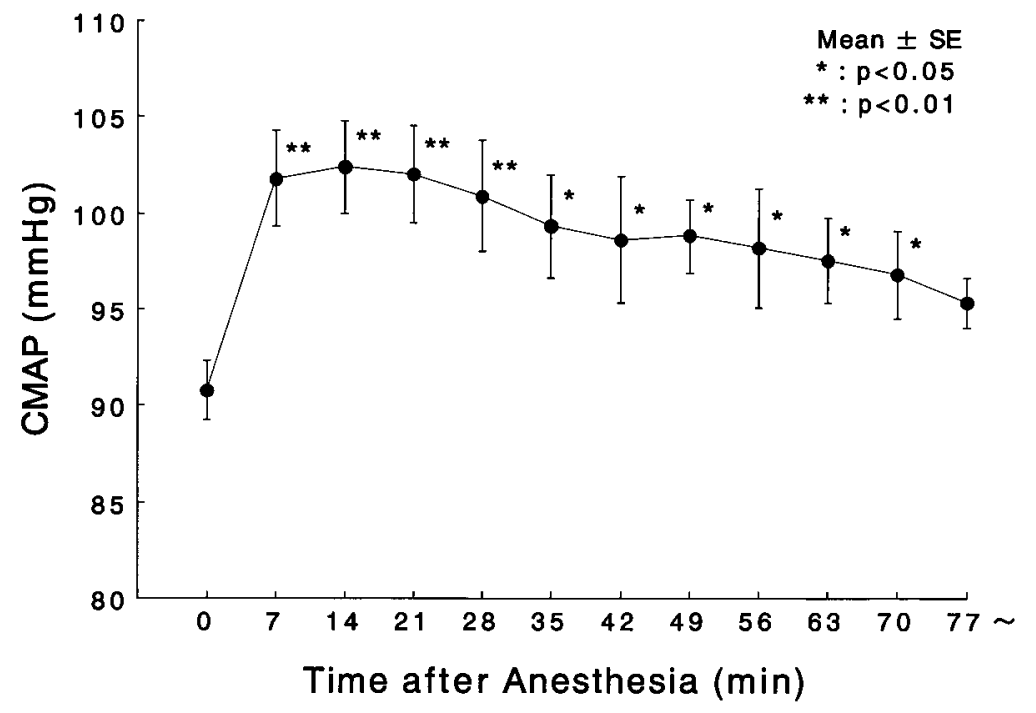

Fig. 4. Time-dependent change in CMAP after intravenous administration of pentobarbital sodium at a dose of $25.0 \mathrm{mg} / \mathrm{kg}$. CMAP was calculated as an average in MAP for $30 \mathrm{sec}$ before rapid mild hemorrhage. CMAP at 0 and 77- min were determined from the MAP response to rapid hemorrhage averaged 8 and 4 times under conscious and anesthetized (from 77 to $98 \mathrm{~min}$ after the anesthesia) conditions, respectively. Significant difference between the preanesthetic and anesthetized states at each time was examined by Tukey's multi-comparison test. 


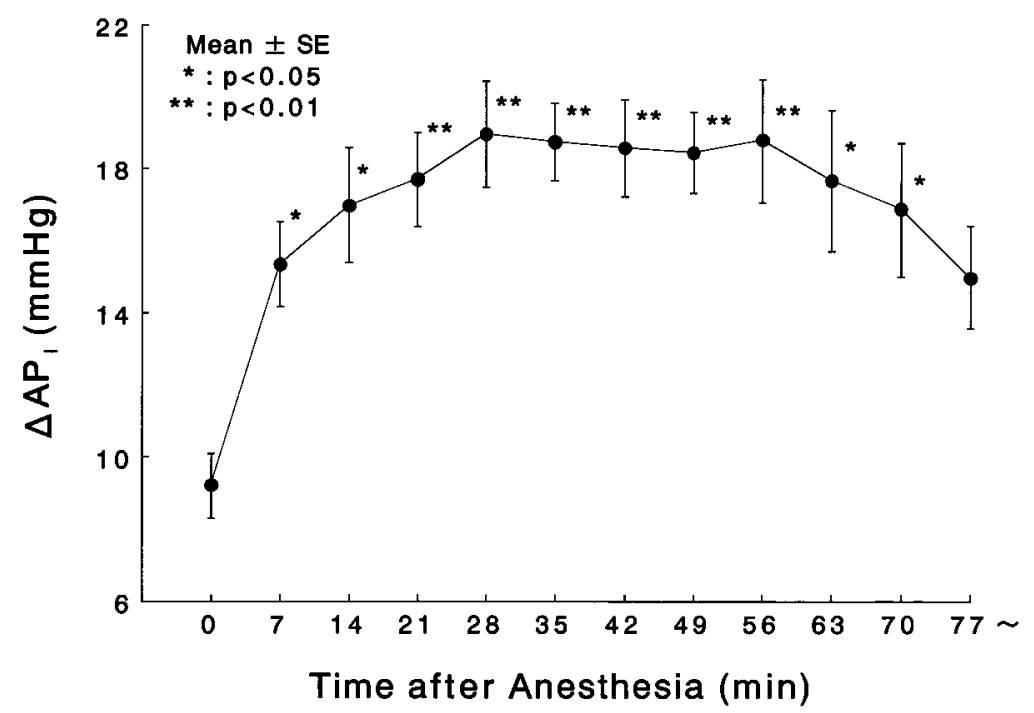

Fig. 5. Time-dependent change in $\Delta \mathrm{AP}_{\mathrm{I}}$ after intravenous administration of pentobarbital sodium at a dose of $25.0 \mathrm{mg} / \mathrm{kg} . \Delta \mathrm{AP}_{\mathrm{I}}$ at 0 and $77-\mathrm{min}$ were determined from averaged MAP response to rapid hemorrhage 8 and 4 times under conscious and anesthetized (from 77 to 98 min after the anesthesia) conditions, respectively. Significant difference was tested as shown in Fig. 4.

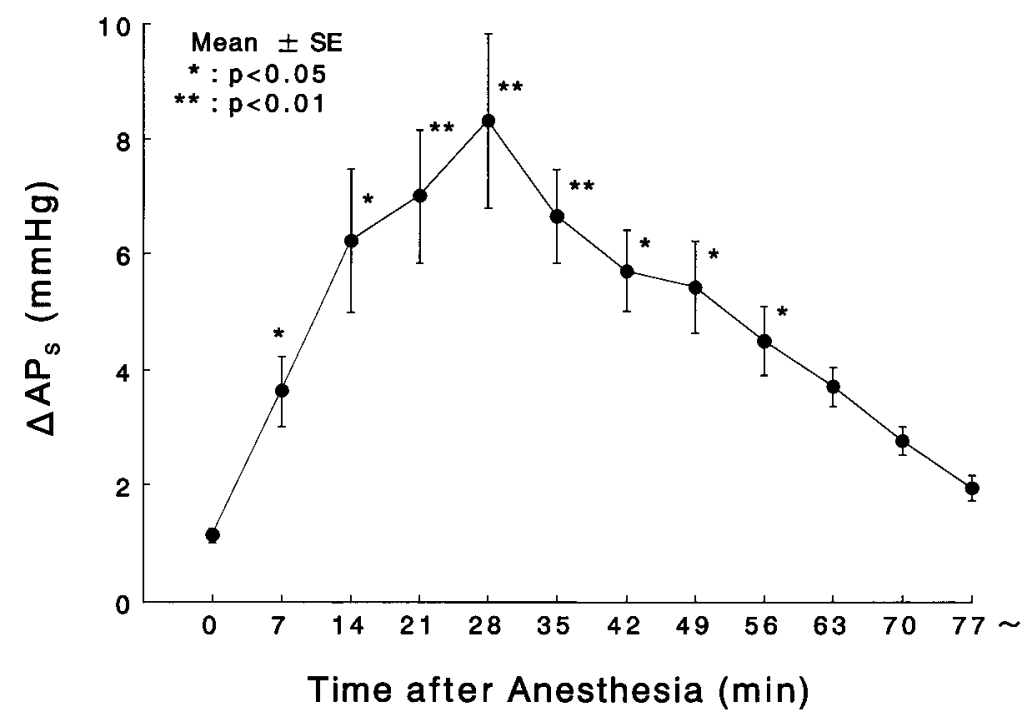

Fig. 6. Time-dependent change in $\triangle \mathrm{AP}_{\mathrm{S}}$ after intravenous administration of pentobarbital sodium at a dose of $25.0 \mathrm{mg} / \mathrm{kg} . \Delta \mathrm{AP}_{\mathrm{S}}$ at 0 and $77-\mathrm{min}$ were determined from averaged MAP response to rapid hemorrhage 8 and 4 times under conscious and anesthetized (from 77 to $98 \mathrm{~min}$ after the anesthesia) conditions, respectively. Significant difference was tested as shown in Fig. 4.

an increase in AP in acute animal studies, other investigators $[4,8,14,24]$ have reported that the AP level was not significantly different from the control level. Fray et al. [4] and Zimpfer et al. [24] suggested that this difference would result from that between the animals used i.e., trained or untrained, and that excitement and surgical trauma in introducing an untrained animal to general anesthesia would also be responsible for a 


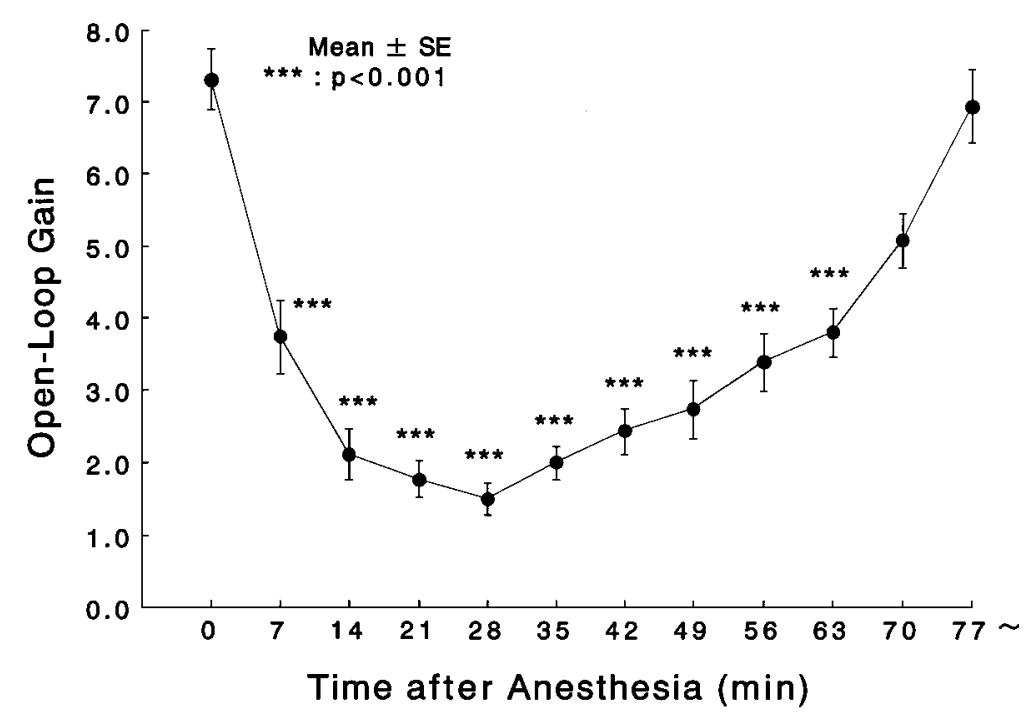

Fig. 7. Time-dependent change in $\mathrm{G}$ after intravenous administration of pentobarbital sodium at a dose of $25.0 \mathrm{mg} / \mathrm{kg}$. G at 0 and $77-$ min were determined from averaged MAP response to rapid hemorrhage 8 and 4 times under conscious and anesthetized (from 77 to 98 min after the anesthesia) conditions, respectively. Significant difference was tested as shown in Fig. 4.

rise in the AP level. The effects of pentobarbital sodium on the cardiovascular regulatory system have been studied to investigate the mechanism of circulatory action of the anesthetics. Pentobarbital sodium has been shown to have the suppressing effects on the sympathetic $[5,16,21,22]$ or parasympathetic nervous system $[5,19]$, which involved the reduction in baroreflex function.

The effect of pentobarbital sodium on the baroreflex system has often been evaluated with AP or HR responses to cardiovascular stimulus, e.g., hemorrhage. Vatner and Braunwald [23] showed a difference in AP control function after hemorrhage in the conscious and anesthetized states. Cox and Bagshaw [2] demonstrated that pentobarbital sodium modified cardiovascular responses to carotid hypotension. On the other hand, Hosomi and Sagawa [8] reported no effect of pentobarbital anesthesia on the baroreflex control capacity of AP after the hemorrhage of $10 \%$ of blood volume within 20-30 sec in dogs. Zimpfer et al. [24] carried out a hemorrhage experiment with the same protocol as that of Hosomi and Sagawa [8] to reconfirm the effect of pentobarbital anesthesia, but it failed to be consistent with their conclusions. This discrepancy has been controversial.
Thus, the effect of pentobarbital anesthesia on the baroreflex system is at variance among investigators. The disparity in the cardiovascular effects of pentobarbital sodium would arise from the difference in the assessment of baroreflex function or that in the process of recovery from the anesthesia, in addition to the differences in dosage, species, age and other experimental conditions. The amount of hemorrhage and analysis of cardiovascular response to the hemorrhage seem to be important factors in precisely assessing the cardiovascular control capacity of the baroreflex system.

The AP control capacity of the baroreflex system has often been evaluated with a $G$ value determined from AP responses to hemorrhage by the application of the control theory to the system [15]. Most investigators have employed massive hemorrhage to determine $\mathrm{G}$ in dogs. Massive hemorrhage causes a large decrease in blood volume, resulting in abnormal blood distribution to the organs and a decrease in systemic blood pressure. In some cases, the animals could show shock-like manifestations. The hypovolemia could affect humoral or hormonal cardiovascular regulatory mechanisms and cause hypoxia, acidosis, and so forth. Hosomi et al. [10] previously assessed the time-dependent changes in the AP control capacity of the baroreflex system after 
massive hemorrhage $(5 \mathrm{ml} / \mathrm{kg}$, body weight) with the open-loop gain determined by rapid mild hemorrhage. The control capacity of the baroreflex AP control system progressively decreased with time after massive hemorrhage and tended to recover somewhat by 110 min after hemorrhage. Therefore, the evaluation of the baroreflex AP control capacity under the hypovolemic conditions due to massive hemorrhage, including estimation of time-dependent effects of anesthetics, is not considered to show the precise control capacity of the system because massive hemorrhage must be a parametric forcing which modifies the normal characteristics of the baroreflex AP control system [6-9, 16-18].

On the other hand, rapid mild hemorrhage is the withdrawal of a small amount of blood to allow us to do a precise physiological evaluation of baroreflex function $[6,7,9-11,13]$. The MAP fall immediately after rapid mild hemorrhage $\left(\triangle \mathrm{AP}_{\mathrm{I}}\right)$ is a transient fall in AP due to a small decrease in blood volume in the aorta. $\Delta \mathrm{AP}_{\mathrm{I}}$ is observed in animals whose AP feedback baroreflex control system has been completely eliminated [9]. The baroreflex system operates at least about $2 \mathrm{sec}$ after the application of external disturbances, such as hemorrhage, to the system [20]. Immediately after the rapid mild hemorrhage, the baroreflex system does not function [20]. These facts suggest that $\Delta \mathrm{AP}_{\mathrm{I}}$ is in major part determined by cardiovascular mechanical properties, such as distensibility of the aortic wall, capacity of the aorta, rate of filling the aorta with blood, rate of draining blood from the aorta to the peripheral vessels, and so forth. The baroreflex system functions most powerfully 1-2 min after the hemorrhage [9]. The MAP fall $\left(\Delta \mathrm{AP}_{\mathrm{S}}\right)$ is a steady-state error in the system which is observed in each kind of hemorrhage experiment irrespective of the hemorrhage volume. $\Delta \mathrm{AP}_{\mathrm{I}}$ and $\Delta \mathrm{AP}_{\mathrm{S}}$ must be a physiological test input and an output in the baroreflex system, respectively. Therefore, the baroreflex system could be considered to be a proportional negative-feedback control system. The input-output relationship determined by rapid mild hemorrhage can estimate the normal characteristics of the baroreflex system. In previous reports, the values for overall open-loop gain of the baroreflex system estimated by method of the rapid mild hemorrhage were 7.4 and 7.8 in anesthetized rabbits [11] and dogs [10] with pentobarbital sodium in intravenous doses of 27.5 and $35.0 \mathrm{mg} / \mathrm{kg}$, respectively. These suggest that there is no remarkable difference between the two species in the overall AP control capacity of the baroreflex system in this dose range.

We previously compared the values for CMAP, $\Delta \mathrm{AP}_{\mathrm{I}}$, $\triangle \mathrm{AP}_{\mathrm{S}}$ and $\mathrm{G}$ in the conscious state with those determined 60-90 min after the anesthesia in normal and Watanabe heritable hyperlipidemic (WHHL) rabbits, since the MAP responses were unstable for about 60 min after the onset of the anesthesia [13]. The G value resulted in showing no significant difference between the conscious and anesthetized conditions in either rabbit group. This suggests that the effect of pentobarbital anesthesia on the baroreflex system is time-dependent. In the present study, there were no significant differences in these values between the conscious and anesthetized states $77 \mathrm{~min}$ after the onset of the pentobarbital anesthesia. This means that the overall AP control capacity of the baroreflex system has almost recovered from the effect of pentobarbital anesthesia $77 \mathrm{~min}$ after the anesthesia at a dose of $25.0 \mathrm{mg} / \mathrm{kg}$. The time-dependent change in CMAP after the anesthesia did not parallel those of $\Delta \mathrm{AP}_{\mathrm{S}}$ and $\mathrm{G}$ though there were no statistical significant differences between their preanesthetic level and that $77 \mathrm{~min}$ after the anesthesia. CMAP and $\triangle \mathrm{AP}_{\mathrm{I}}$ rose about 1.1 and 2.1 fold of the preanesthetic value at maximum, respectively, whereas $\triangle \mathrm{AP}_{\mathrm{S}}$ and $\mathrm{G}$ showed a larger increase and decrease about 7.4 and 0.2 fold of the preanesthetic value at maximum, respectively, in comparison to changes in CMAP and $\triangle \mathrm{AP}_{\mathrm{I}}$. Although the site of action of pentobarbital sodium was not investigated in the present study, we can suppose that this anesthetic, restricted to circulatory function, would affect both the peripheral mechanical function of the heart and blood vessels and the central and peripheral nervous systems from the baroreceptor to the sympathetic and parasympathetic nerves because of changes in both the input and output values to the system caused by the anesthesia. The complicated central and peripheral actions of pentobarbital sodium would cause differences in maximal changes in $\triangle \mathrm{AP}_{\mathrm{I}}, \Delta \mathrm{AP}_{\mathrm{S}}$ and $\mathrm{G}$ in response to rapid mild hemorrhage, which would in part be responsible for the disparity in the recovery rates of these variables from the effects of anesthesia.

We could compromise one of the discrepancies in the effect of pentobarbital anesthesia on the baroreflex AP control system in consideration of the time-depend- 
ent change in the overall AP control capacity of the baroreflex system. The differences in the cardiovascular effects of pentobarbital sodium due to those in species, dosage and method of administration, e.g. intravenous, intraperitoneal or subcutaneous, should be elucidated in future. In evaluating cardiovascular function under anesthetized conditions, consideration of the time-dependent effect of anesthetics, as well as differences in species, dosage, animal conditions, sensitivity to anesthetics, and so forth could provide some useful information on the cause of variation in the measured cardiovascular parameters. Moreover, it is also important to reconfirm whether cardiovascular stimulus and the method used in analyzing the cardiovascular response to the stimulus precisely represent physiological regulatory function of the cardiovascular system or not.

\section{Acknowledgment}

We express our heartfelt thanks to the late Dr. H. Hosomi, a former professor of Kagawa Medical School for his kind suggestions and encouragement in the present study.

\section{References}

1. Bedran-de-Castro, M.T., Faras, V.M., and Krieger, E.M. 1990. Influence of general anesthesia on baroreflex control of circulation. Brazil. J. Med. Biol. Res. 23: 1185-1193.

2. Cox, R.H. and Bagshaw, R.J. 1979. Influence of anesthesia on the response to carotid hypotension in dogs. Am. J. Physiol. 237: H424-H432.

3. Duan, V.F., Winters, R.W., McVabe, P.M., Green, E.J., and Schneiderman, N. 1994. Basal and reactive plasma catecholamine levels under stress and anesthesia in rabbits. Physiology and Behavior 56: 577-583.

4. Fray, J.C.S., Siwek, L.G., Strull, W.M., Steller, R.N., and Wilson, J.M. 1976. Influence of dietary sodium on renin activity and arterial pressure during anesthesia. Am. J. Physiol. 231: 1185-1190.

5. Halinen, M.O., Hakumaki, M.O., and Sarajus, H.S. 1978. Suppression of autonomic postganglionic discharges by pentobarbital in dogs, with or without endotoxemia. Acta Physiol. Scand. 104: 167-174.

6. Hosomi, H. 1978. Overall characteristics of arterial pressure control system studied by mild hemorrhage. Am. J. Physiol. 234: R104-R109.

7. Hosomi, H. 1978. Unstable state of the arterial pressure control system after a mild hemorrhage. Am. J. Physiol. 235: R279-R285.

8. Hosomi, H. and Sagawa, K. 1979. Effect of pentobarbital anesthesia on hypotension after $10 \%$ hemorrhage in the dog. Am. J. Physiol. 236: H607-H612.

9. Hosomi, H. and Yokoyama, K. 1981. Estimation of openloop gain of canine arterial pressure control system by a new method. Am. J. Physiol. 240: H832-H836.

10. Hosomi, H., Baba, M., and Morita, H. 1981. Timedependent changes in open-loop gains of baroreflex systems after massive hemorrhage. Jpn. J. Physiol. 31:705-715.

11. Hosomi, H., Katsuda, S., Morita, H., Nishida, Y., and Koyama, S. 1986. Interactions among reflex compensatory systems for posthemorrhage hypotension. Am. J. Physiol. 250: R944-R953.

12. Jung, R., Bruce, E.N., and Katona, P.G. 1989. Tonic and baroreflex effects on arterial pressure and ventilation of pentobarbital and nicotine on the rat ventral medullary surface. Brain Res. 485: 399-402.

13. Katsuda, S., Hosomi, H., Shiomi, M., and Watanabe, Y. 1992. Impaired baroreflex control of arterial pressure in WHHL rabbits. J. Vet. Med. Sci. 54: 983-987.

14. Manders, W.T. and Vatner, S.F. 1976. Effects of sodium pentobarbital anesthesia on left ventricular function and distribution of cardiac output in dogs with particular reference to the mechanism for tachycardia. Circ. Res. 39: 512-515.

15. Millhorn, H.T.Jr. 1966. The application of control theory to physiological systems, Vol. 1, 1st ed., B.W. Saunders, London.

16. Morita, H., Nishida, Y., Uemura, N., and Hosomi, H. 1987. Effect of pentobarbital anesthesia on renal sympathetic nerve activity in the rabbit. J. Autonom. Nerv. Sys. 20: 57-64.

17. Morita, H., Nishida, Y., Motochigawa, H., Watanabe, Y., and Hosomi, H. 1988. Depressed baroreflex control of renal nerve activity in conscious WHHL rabbits. Cardiovasc. Res. 22: 679-685.

18. Morita, H., Nishida, Y., Motochigawa, H., Uemura, N., Hosomi, H., and Vatner, S.F. 1988. Opiate receptormediated decrease in renal nerve activity during hypotensive hemorrhage in conscious rabbits. Circ. Res. 63: 165-172

19. Murthy, V.S., Zager, M.E., Vollmer, R.R., and Schmidt, D.H. 1982. Pentobarbital-induced changes in vagal tone and reflex vagal activity in rabbits. Europ. J. Pharmacol. 84: 41-50.

20. Scher, A.M. and Young, A.C. 1963. Servoanalysis of carotid sinus reflex vasomotor responses effects on peripheral resistance. Circ. Res. 12: 152-162.

21. Stornetta, R.L., Guyenet, P.G., and McCarty, R.C. 1987. Autonomic nervous system control of heart rate during baroreceptor activation in conscious and anesthetized rats. J. Autonom. Nerv. Sys. 20: 121-127.

22. Teranishi, Y. and Iriuchijima, J. 1992. Sympathetic vasoconstrictor tone induced by pentobarbital anesthesia in hindquarters of rats. Jpn. J. Physiol. 42: 171-178.

23. Vatner, S.F. and Braunwald, E. 1975. Cardiovascular control mechanisms in the conscious state. New Eng. J. Med. 293: 970-976.

24. Zimpfer, M., Manders, W.T., Barger, A.C., and Vatner, S.F. 1982. Pentobarbital alters compensatory neural and humoral mechanisms in response to hemorrhage. Am. J. Physiol. 243: H713-H721. 